Document downloaded from:

http://hdl.handle.net/10251/134996

This paper must be cited as:

Sendra, S.; Parra-Boronat, L.; Lloret, J.; Tomás Gironés, J. (2018). Smart system for children's chronic illness monitoring. Information Fusion. 40:76-86.

https://doi.org/10.1016/j.inffus.2017.06.002

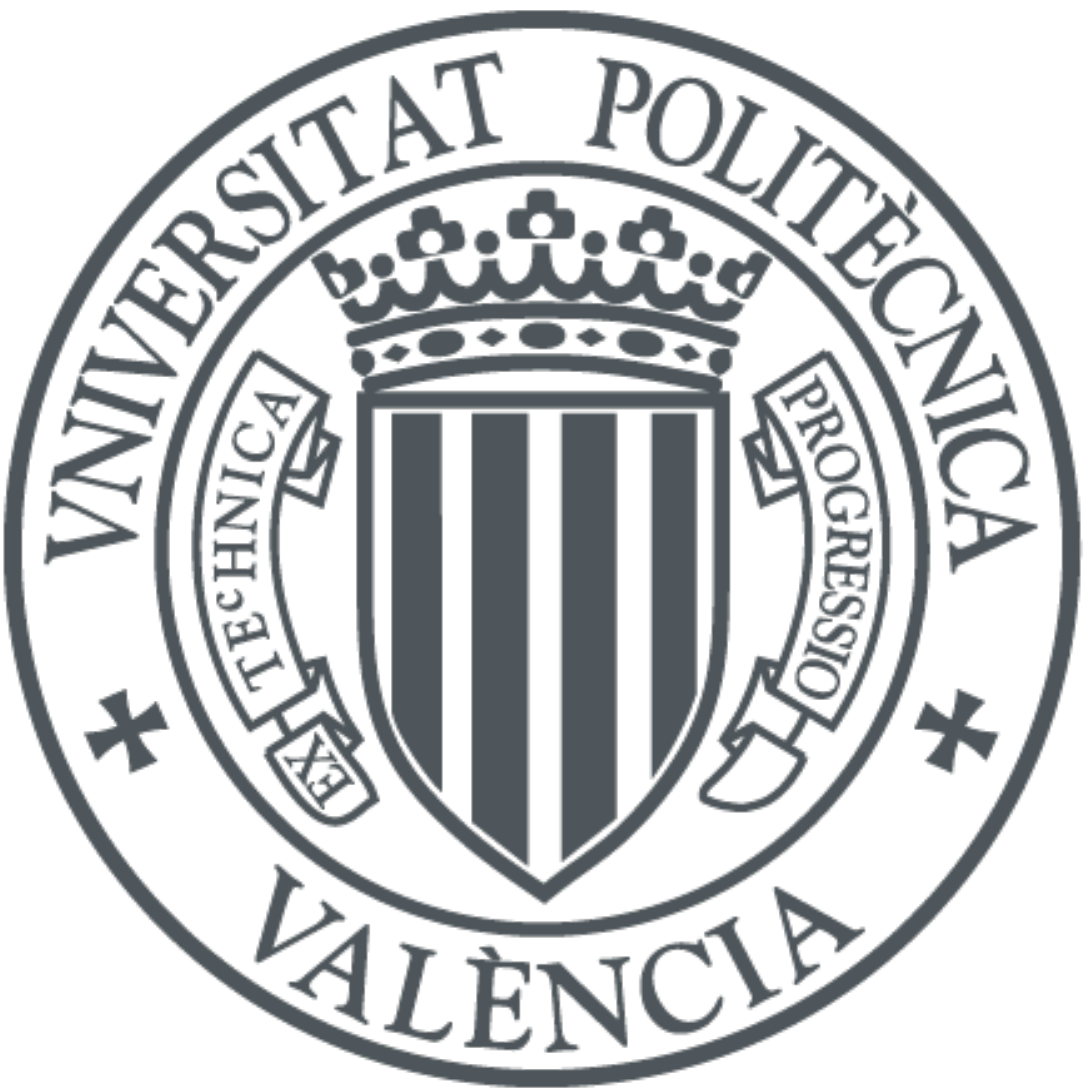

The final publication is available at

https://doi.org/10.1016/j.inffus.2017.06.002

Copyright Elsevier

Additional Information 


\title{
Smart System for Children's Chronic Illness Monitoring
}

\author{
Sandra Sendra ${ }^{1,2}$, Lorena Parra ${ }^{2}$, Jaime Lloret $^{2}$, Jesus Tomás ${ }^{2}$ \\ ${ }^{1}$ Dept. of Signal Theory, Telematics and Communications Department (TSTC), Universidad de Granada, \\ Spain \\ ${ }^{2}$ Instituto de Investigación para la Gestión Integrada de Zonas Costeras (IGIC), Universitat Politecnica de \\ Valencia, Spain
}

ssendra@ugr.es, loparbo@doctor.upv.es, jlloret@dcom.upv.es, jtomas@upv.es

\begin{abstract}
Sick children need a continuous monitoring, but this involves high costs for the government and for the parents. The use of information and communication technologies (ICT) jointly with artificial intelligence and smart devices can reduce these costs, help the children and assist their parents. This paper presents a smart architecture for children's chronic illness monitoring that will let the caregivers (parents, teachers and doctors) to remotely monitor the health of the children based on the sensors embedded in the smartphones and smart wearable devices. The proposed architecture includes a smart algorithm developed to intelligently detect if a parameter has exceeded a threshold, thus it may imply an emergency or not. To check the correct operation of this system, we have developed a small wearable device that is able to measure the heart rate and the body temperature. We have designed a secure mechanism to stablish a Bluetooth connection with the smartphone. In addition, the system is able to perform the data fusion in both the information packetizing process, which contributes to improve the protocol performance, and in the measured values combination, where it is used a stochastic approach. As a result, our system can fusion data from different sensors in real-time and detect automatically strange situations for sending a warning to the caregivers. Finally, the consumed bandwidth and battery autonomy of the developed device have been measured.
\end{abstract}

Keywords—Smart algorithm; chronic diseases; child monitoring; ehealth; wearable devices.

\section{INTRODUCTION}

In the last four decades, the number of so-called chronic diseases is on the rise among children in USA [1]. While some illnesses as different type of cancers or congenital heart disease have higher rates of survival and the infections have diminished, the chronic diseases have higher impact in young people [2]. Researchers as Van Cleave et al. in [3] studied several chronic diseases such as obesity, asthma, allergic condition, epilepsy and hyperactivity disorder in more than 5000 children younger than 8 years old since 1988 to 2006. As some background data, in Australia up to $70 \%$ of the disease burden is related to chronical diseases and it is expected to reach the $80 \%$ in 2020 [4]. Moreover, the $23.8 \%$ of male children and 22.6 of females of developed countries have obesity, in developing countries the values are $8.1 \%$ and 8.4\% respectively [5]. Furthermore, children with epilepsy have 5.3 times higher probability of dying than children without epilepsy [6]. In these cases, the need of a smart chronic illness monitoring system is clearly justified.

By definition the chronic diseases are permanent and nonreversible illnesses, which may require supervision and care by physicians [7]. This supervision is especially important when the chronic disease is suffered by a child. The self-management is very important and many countries promote different programs [4]. Self-management in children is necessary to ensure treatment efficacy, however it supposes a challenge for children, families and physicians [8]. The main aim of the self-management is to increase the life quality of the patient by improving the self-care and facilitating the interaction doctor-patient [4].

The use of information and communication technologies (ICT), jointly with artificial intelligence techniques and smart devices, can be an essential part of the self-management plans. They have been especially successfully used to improve human health [9]. Smart devices such as smartphones have multimedia sensors that can help to monitor the vital signs like heartbeat rate, respiratory rate and respiratory alterations among others [10]. By one side, the accelerometers, gyroscope and GPS can help to monitor the position, movement and detect possible falls [11]. By the other side, wearable devices offer other options such as detection of seizures in epileptic patients [12] or allow the posture and activity recognition for obesity treatment [13]. 
The advantages of using smart devices for monitoring children with chronical diseases are many and varied. From the point of view of the children, the possibility of reducing their dependence on hospitals or constant supervision by the caregivers, gives them the chance on enjoy their childhood. This will suppose an increase of their quality of life from the physical and psychological point of view. Moreover, there is a possibility to improve the treatment and avoid dangerous episodes depending on the disease. From the point of view of the caregivers or the parents, it will allow them to reduce the pressure that supposes the continuous monitoring of their child and reduces also the time they spend in the hospitals. For the caregivers, this system allows real-time monitoring of all of their patients remotely. It will promote the better attention of the patient when the children go to the hospital. For the hospitals, the self-management means a considerable economic saving on personal and infrastructure terms. Finally, to gather such amount of data form patients with chronic diseases will promote an advance of our knowledge. The security and fault tolerance on networks for eHealth is extremely important as it has been shown in [14].

Bergström et al. in [15] conclude that $62 \%$ of children dying of asthma happens because of undertreatment. The real time and remote monitoring by the doctors in conjunction with smartphone applications for the children and its caregivers can prevent some of this death. Germann et al. found that the patients with obesity that self-monitoring most days loss more weigh that children that do not [16].

It is expected that, the innovation in the area of mobile devices and sensor technologies will reduce the health management costs. This can even go ahead to make the mobile-health (m-health) a reality [17]. There are several related work that show the use of ICT and especially the use of sensors to monitor and take care of ill people such as [18], [19] and [20]. To the best of our knowledge, no other work is focused on the use of eHealth systems for improving children's life or assisting them to be monitored when they are sick. This paper is focused on bringing a solution to this problem.

In this paper, we present a smart system for children's chronic illness monitoring. Using the data gathered by the smartphone sensors and data from wearable devices, the child can be remotely monitored by the caregivers (parents, teachers and doctors) [21]. A smart algorithm, which is capable to send the data to the caregivers, store it in database and label the alarms as positive or false positive after it has been the validated (or not) by the physician is shown. Data fusion [22] [23] is presented by using a stochastic approach, as a result our system can fusion data from the different sensors in real-time and detect automatically the alarm situations in order to send a warning message to the caregivers. This system can be adapted for monitoring different chronic diseases by just adding the needed sensors. In our study case, the included sensors facilitate the monitoring of asthma disease. As it has been aforementioned, asthma can cause the death in children [15]. The vital signs that will be monitored are the following ones: heart rate, breathing rate, oxygen saturation and activity index. This vital signs correspond from one side to the effects of asthma, reduction of oxygen saturation and alteration of breathing and heart rate pattern during an asthma attack. The detection of changes in heart rate can be very useful to define preceding symptoms of some illness and emotional states including fear [24]. By the other side, the main medicines for asthma attack treatment can cause side effects as increase the heart rate and the child activity. Other sensors like temperature sensor and sweeting sensors are included to detect other abnormal situations.

The remainder of the article is organized as follows. We study some related works present in the literature in Section II. In Section III, we present our system, the proposed architecture, the smart algorithm, the packet exchange and the architecture for children monitor. The stochastic approach for multi-sensor fusion decision algorithm that we implement is presented in Section IV. Section V details the scenario of our test bench and the obtained results in terms of battery consumption and consumed bandwidth. Finally the conclusions are shown in Section VI.

\section{RELATED WORK}

In this section the state of art of eHealth systems based on the use of sensors for patient monitoring is shown. They have become a hot topic in the last years. Ambient Assisted Living (AAL) systems are able to collect data with sensors, store it in a database (DB) and use it for different purposes [25]. They can be composed by sensors embedded in the smartphone or use wearable sensors forming a Wireless Sensor Network (WSN) [26].

Now, we are going to detail some of the current works that use the sensors of the smartphone for ehealth monitoring.. A smart collaborative system was proposed by Sendra et al. in [17]. The system used the sensors of the smartphone for monitoring disabled or elderly people. Different sensors of smartphones can be used by the system as microphone, gyroscope, Global Positioning System (GPS), accelerometer and light sensors among others. The system was capable to obtain data for the monitored person and compare it with the data form the neighbors and what is happening with the environment. Comparing the data the system can take different decisions and evaluate if a person falls down or leaves a group. Different tests have been developed using the sensors of different smartphones in order to evaluate the different behaviors of different users. Moreover, the system was able to generate an alarm if an abnormal behavior in the person was detected. 
Other system focused on fall detection was presented by Dai et al. in [27]. Authors presented PerFallD, a pervasive fall detection system that employed the sensors of the smartphones to identify the falling behavior. They presented two different algorithms that detect the falls according for smartphones with and without magnetic accessories. The system obtained the data from the accelerometer of the smartphones and used the $\mathrm{X}, \mathrm{Y}$ and $\mathrm{Z}$ acceleration. The smartphone was placed at the position of the chest, waist and thigh during the tests. Tests were done using mannequins and with real people. The employed smartphones were an Android G1 and a magnetic accessory in the leg. Authors compared the performance of their system with a commercial one. Their system presented higher accuracy and lower energy consumption than the commercial one.

A system for frailty monitoring was proposed by Bravo et al. in [28]. The system was focused on rehabilitation process and daily activity monitoring. Their system was able to diagnose and monitor frailty. The accelerometer embedded in the smartphone was used to obtain information about the movement of the person. Different tests were done with elderly people in a residence to study the walking, siting up and siting down behavior of people with and without frailty. The acceleration values in $\mathrm{X}, \mathrm{Y}$ and $\mathrm{Z}$ axis of a sensor were used to diagnose. A smooth process must be applied to the data before using it for monitoring or diagnose. The system did not need any explicit interaction of the patient with the smartphone. The system was focused on rehabilitation process and daily activity monitoring.

In [29], Ketabdar and Lyra proposed a system for monitoring physical activities of elderly or disabled people by using the data of the sensor of the smartphone. The data of the acceleration in $\mathrm{X}, \mathrm{Y}$ and $\mathrm{Z}$ axis were used to recognize the activity of the user after being processed. Authors performed a test where 320 activity instances with 4 subject users were recorded and analyzed. From the 320 records, 208 of them were used to train the system and the other 112 to test it. Different behaviors were selected for the system as walk, run, rest and no activity. The system presented an accuracy of 92.9\%. Moreover, the authors showed the visualization of the ActivityMonitor screen, the statistics or the warnings from their developed software.

In [30] Lee et al. presented a system that allows elderly people and chronically ill patients monitoring. Using this system patient can stay independently at home and avoid hospitalization during long periods of time. The system was implemented in a smartphone and can detect a fall and communicate the data to a server using TCP/IP via WIFI technology. Their system was composed by a smartphone, HTC Desire A8181, which gathered data from the 3-axial accelerometer. The system also included a remote panic button.

Now, the related work where authors use wearable sensors in addition to smartphones are presented. Those systems were based on the use of wireless body area sensor network (WBAN). Lee and Chung in [31] presented wearable smart shirt for ubiquitous health and activity monitoring. Their system allowed measuring electrocardiogram and acceleration signals for continuous and real time health monitoring. The physiological data, electrocardiogram, and the activity data, acceleration, gathered by the sensors were transmitted from the node in an ad-hoc network to a base-station and server. Authors developed a test to evaluate the performance of the system. The smart shirt was used during different activity patterns as running, walking and resting. The electrocardiogram and the Y-axis acceleration were the most representative data to identify the activity of the user.

A data relaying scheme for healthcare monitoring of mobile patients at home was presented by González-Valenzuela et al. in [32]. Their system was based on a WBAN and it was capable to obtain data of the vital signs of the patients. The aim of their system was to maintain the patients at their homes under non-critical conditions. Authors presented the architecture and protocols of their proposal. The system was composed by four sensors placed in the upper left-arm, the left wrist, the waist and the ankle. Authors showed the relay utilization of the sensors under different activity patterns and the lost packets of each sensor.

Aminian et al. in [33] presented a monitoring system based on a WBAN, which was able to sense vital signals of patients during a hospitalization. Some of the measured parameters were the heart rate and the blood pressure among others. WBAN was composed by four different sensors placed along the body of the patient. The system can send the data to a base station thought a Wireless Multi-Hop Relay Node. A system of alerts was developed. It advices to the doctor and the patient in the case of any of the vital signs presents abnormal values. The authors presented the coverage of different nodes along the hospital, the energy consumption and even the delay on different scenarios. They also compared the performance of their proposal with other proposals. Their proposal presented lower delay that the other ones

A proposal for real time monitoring of patients in indoor and outdoor scenarios was presented by Bourouis et al. in [34]. Authors presented an architecture formed by a star based WBAN, a smartphone, and a GSM tower to transmit the information to the intelligent central server. Their proposal was tested in a Nokia Smartphone N95 with Operating System Symbian v9.2. Authors created Python application for S60 in ICN. The application gathered data form the wearable sensors (heart rate and blood oxygenation) and 
form the phone (accelerometer among others). The communication between the wearable sensors and the smartphone was done with Bluetooth. Authors presented some screenshots of the applications on the phone and the webpage interface at the server. If an abnormal situation was detected, an alert was sent to the medical professionals or to the family. The alert included the location of the patient to facilitate the sending of help.

Similar systems were proposed by Gay and Leijdekkers in [35]. They were focused on monitoring the wellbeing of high risk cardiac patients. Postolache et al. in [36] included the use of a wrist-worn band that measured the vital sings with activity recognition and a smartphone application. Leijdekkers et al in [37] presented a system for patient monitoring using smartphones, wireless sensors, web servers and IP webcams. Lorincz et al. in [38] showed a system for high-fidelity motion analysis. A smart communication architecture focused on monitoring elderly people in indoor scenarios was shown by Lloret et al. in [39].

No one of the revised systems is specially designed and developed for children monitoring. There are many proposals for elderly people (such as [38], [39] and [40]), and for disabled people such as [41]. However no specific architecture or protocol found in the related literature is focused on the use of eHealth for children. The few examples that can be found about eHealth with pediatric purposes are web-based services. Moreover, the telephone call systems for pediatric asthma control [42], [43] need the interaction of parents. Our proposal will no need the direct interaction of parents or other caregivers to trigger the system in order to send data or generate alarms. In our proposal, alarms are activated by the data gathered (based on normal or abnormal data).

\section{PROPOSED ARCHITECTURE}

This section presents the proposed architecture as well as the smart algorithm used to take measurements from devices and send them to the parents and physicians.

\section{A. Architecture}

In order to bring a solution to our problem, the designed system consists of three main parts: (1) the mobile application as a local server installed in the smartphone of the caregivers (parents or teachers), (2) the children's smart device that collect the data thanks to the embedded sensors, such as accelerometer, microphone, GPS, camera, etc. We can also consider wearable device other devices such as smartwatches or chest bands that usually communicate with a smartphone using Bluetooth. Finally, this architecture includes a (3) central data base which is located in the hospital or health center. The wireless connection between (1) and (2) can be performed using Wi-Fi or 3G/4G technology. Fig. 1 shows the proposed architecture to smartly monitor the activity and signs of the children with chronic illnesses.

The mobile application, also called the processing unit, (1) installed in the caregivers' smartphone is composed by three components: (i) decision support components, (ii) graphical user interface and (iii) semantic data store. The children's wearable device (2) should be attached in a way that guarantees the child comfort and a wireless connection with the smartphone of the child. The smartphone sends information to a processing unit. The processing unit is the caregivers' mobile application that may be located in the school (during the school time) or at home (when the child is outside the school). The application will automatically switch between them as a function of a preconfigured time table. When an emergency situation is detected, i.e., the system has detected some value out of the specified threshold, the processing unit is sent to the physicians. The connection between (2) and (3) is also performed using 3G/4G technology. Physicians are in charge of validating the data in an emergency situation.

Finally, data taken from the wearable devices and smartphone device is stored in a database. Each medical register is tagged as NORMAL if it corresponds to a measurement where data are OK. It is tagged as POSITIVE if data come from an emergency situation and the physician has validated them as a true emergency event and it is tagged as FALSE POSITIVE when the physician has not validated these data because they do not correspond to an emergency event. These data are used as feedback information to discard further false positive. 


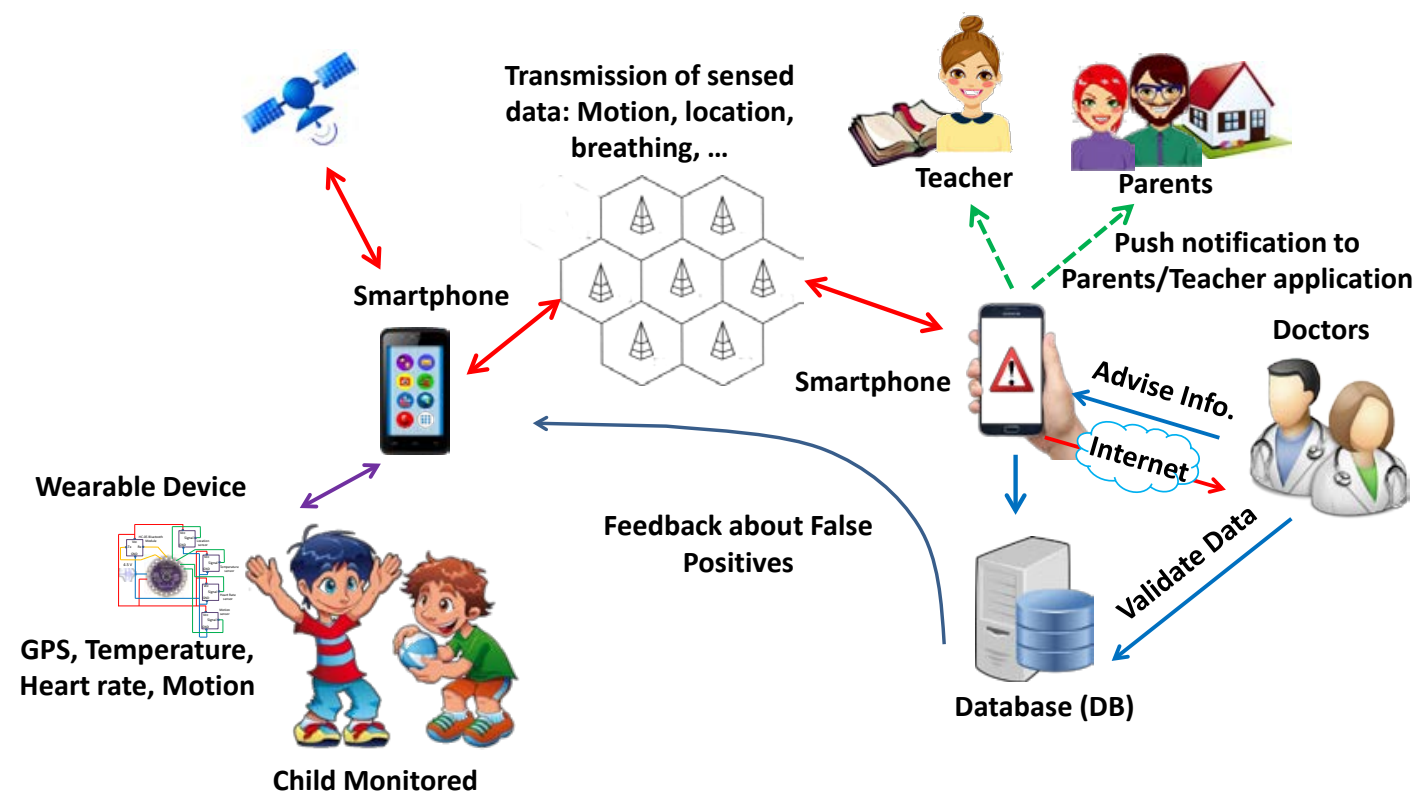

Fig. 1. Proposed architecture for Children's Chronic Illness Monitoring

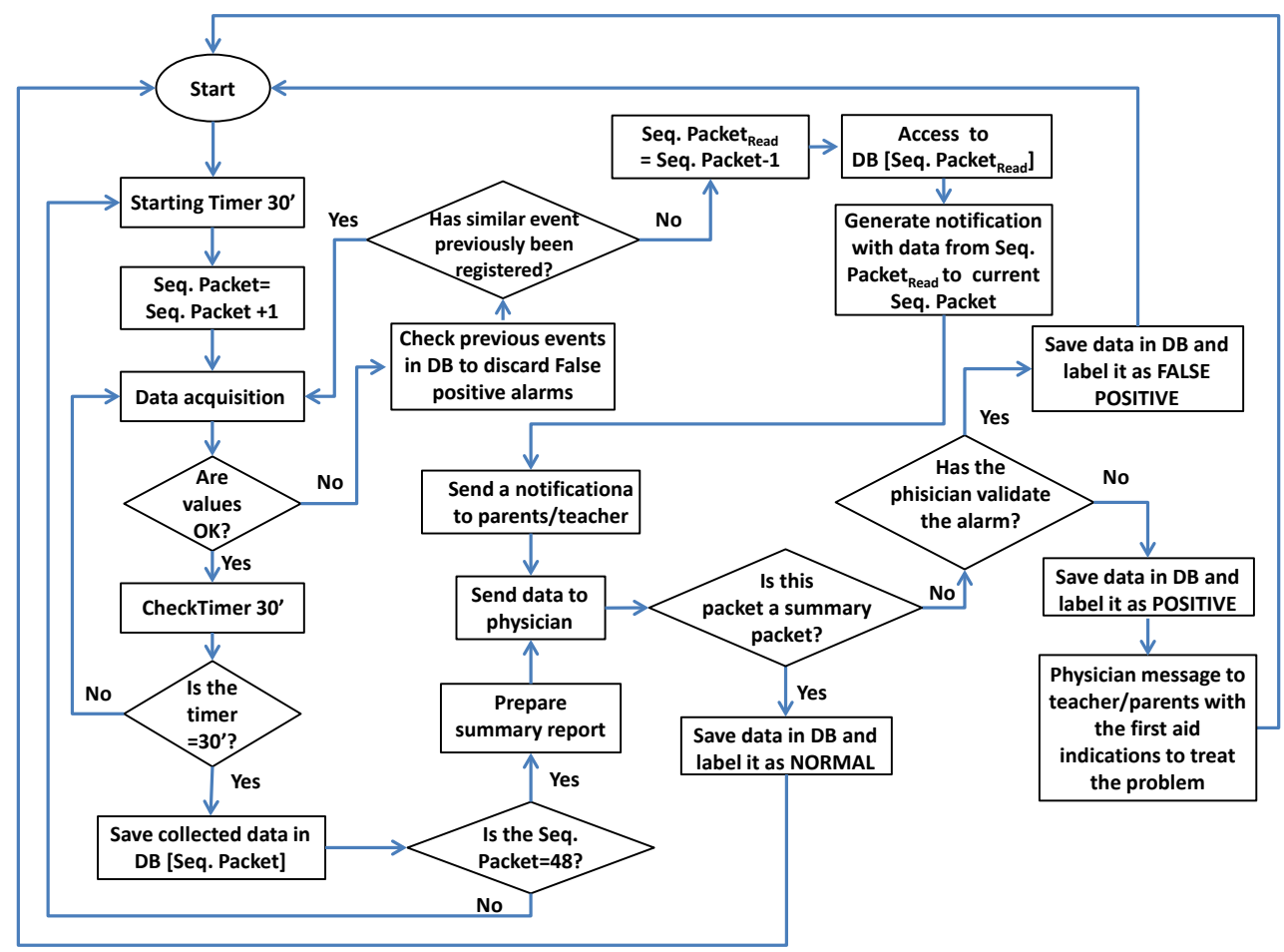

Fig. 2. Smart algorithm for controlling the monitoring device

\section{B. Smart algorithm for Children's Chronic Illness Monitoring}

In a typical functioning scenario, the children's wearable device can be a watch or developed device attached to the hand, a mini-smartphone put in the child's bag when he/she is walking going-toschool/coming-back-home, or in a pocket when playing outside. The signals sent by these devices are received regularly by the processing unit (1) that is responsible of recording in a semantic data store three different types of information: respiration, motion/acceleration and location. It updates the child's health profile in real-time. With this role, the processing unit plays a key feature in our proposed system.

A simple functioning scenario could consist on an outdoor children location system for the parents or teachers. A smart watch like LeapFrog LeapBand could be used to develop our architecture. It is a fitness 
tracker for children that turn the physical activity in a play by giving rewards when some programed challenges are achieved. Another interesting example is Tinitell one smart bracelet. It is equipped with a SIM card and GPS allow locating the children in real time. It can send in real time the child location coordinates to the mobile phone of parents or teachers through Bluetooth. Children playing outside can so be monitored at any time. However, we can develop a new low cost wearable device composed by some specific sensors and we can take profit of the smartphone to send the data.

In some other scenarios, besides the location, other parameters can be taken into account. They can be used to detect functions such as fall detection or respiratory problems. The first function is based on the accelerometers sensor. When a strong acceleration is detected, the application checks (by comparing values) if there is a fall or it is just a false positive (it could happen when the child is jumping or running). If a fall is detected, a notification is then sent to the caregivers (teachers or parents depending on the day moment). If there is not a fall, the application returns back to the situation of falls detection. Respiratory problems detection function is based on the microphone sensor. So, when a strong wheeze is detected, the application checks (by comparing values) if there is a respiratory problem.

To control, monitor and efficiently store the data from the wearable device, we have developed an intelligent algorithm that is able to gather data, analyze them and decide if it is a false positive, a normal packet or the received information is priority data generated because an emergency event has happened.

Fig. 2 shows the smart algorithm for controlling the monitoring device and process the data sensed. As we can see, the system is prepared to be continuously monitoring but it only saves the data in the child's smartphone every 30 minutes. The continuous monitoring is needed to detect sudden events. Each measurement is stored in a DB, and it is identified by a sequence number. Once a day, i.e., when 48 measurements have been stored, the system sends a summary report to the physician in charge of treating this child. This summary report contains the average, minimum and maximum values of the measured parameters. These reports are interesting for observing the evolution in long-term treatments.

When a magnitude exceeds a threshold, the system checks if a similar event has previously been registered in the DB as a false positive. If it is a false positive, the system comes back to the data acquisition phase. If not, it will take the data from the previous sequence packet and the current sequence packet and will generate an alarm message to be send firstly to the teacher, if child is in the school, or to the parents, if the child is at home or outside. After that, this message is transmitted to the physician. In this case, the physician checks and validates the received data. If the data corresponds to an emergency situation, the physician will tag the data as POSITIVE. Otherwise, data will be tagged as FALSE POSITIVE. Finally, when the physician receives the summary report, it is automatically tagged as NORMAL.

On the other hand, when an alarm message is sent to the physician, he/she sends to teacher or parents the first aid indications to treat the problem and mitigate the possible effects on the child, while the emergency services go to the place. The system also has the possibility of remotely advice the teachers and parents.

The next time the system registers a false positive, it will be intelligently detected and it will not send the alarm message to the parents/teachers/physician's smartphones.

\section{StOCHAstic Approach FOR Multi-SENSOR Fusion DeCision AlgORITHM}

Our system must be able to fusion real-time sensors data and detect alarm situations automatically. In order to achieve this goal, we are going to use a general mathematical framework that shows us the multisensor fusion decision algorithm.

\section{A. Stochastic Approach}

In this subsection we explain the mathematical assumptions used in our proposal. We analyse estimation methods from a statistical point of view. Table 1 shows the variables used in the analysis.

Table 1. Mathematical variables used in the model.

\begin{tabular}{cccc}
\hline parameter & description & parameter & Description \\
\hline $\boldsymbol{a}$ & Alarm Indicator & $\boldsymbol{l}$ & Location \\
$\boldsymbol{h}$ & Heart Rate & $\boldsymbol{d}$ & Time of day \\
$\boldsymbol{p}$ & Physic Activity & $\boldsymbol{t}$ & Temperature \\
$\boldsymbol{r}$ & Breathing & $\boldsymbol{T}_{\boldsymbol{i}}$ & Training sample $\left(a_{i}, l_{i}, h_{i}, d_{i}, p_{i}, t_{i}, r_{i}\right)$ \\
\hline
\end{tabular}


The alarm estimation problem can be statistically stated as follows. For simplicity, the true distribution $\operatorname{Pr}(X=x)$ and $\operatorname{Pr}(X=x \mid Y=y)$ are denoted as $\operatorname{Pr}(x)$ which means probability of a variable and $\operatorname{Pr}(x \mid y)$ which means conditional probability of a variable. The model parameters are denoted by $\mathrm{p}()$.

Let $l$ be the location of the children. In our approach, we consider only a finite number of possible locations. For example, in the experimental implementation we differentiate four locations: home, school, outdoor, indoor. Let $d$ be the time of day, taking values from 0 to 23. Let $p$ be the children physic activity. Using the wearable device accelerometer, we can detect the type of activity. Possible values for this feature are: standing, walking, running, cycling, in a car or sleeping. Let $r$ be the abnormal respiration indicator, taking the value 0 when it is not detected and from 1 to 5 depending on the degree of intensity. Finally, $h$ represents the heart rate a $t$ the temperature of the child.

We assume that the generation of an alarm depends only on these six variables. It is shown in equation (1) which represents the prior probability of the alarm:

$$
\operatorname{Pr}(a) \approx \operatorname{Pr}(a \mid l, h, d, p, t, r)
$$

Once this function is estimated, the problem can be formulated to determine the probability of an alarm for a given observation $l, h, d, p, t$ and $r$. Using Bayes' theorem, we can write equation (2). $\operatorname{Pr}(\mathrm{a} \mid l, h, d, p, t, r)$ represents the conditional probability of detecting an alarm $a$ giving the parameters $l, h, d, p, t$ and $r$ :

$$
\operatorname{Pr}(a \mid l, h, d, p, t, r)=\frac{\operatorname{Pr}(a) \operatorname{Pr}(l, h, d, p, t, r \mid a)}{\operatorname{Pr}(l, h, d, p, t, r)}
$$

Where $\operatorname{Pr}(l, h, d, p, t, r \mid a)$ is the likelihood function. It estimates the probability of having the parameters $l, h, d, p, t$ and $r$ knowing that an alarm has occurred. The denominator in equation (2) does not depend on the variable $a$. Therefore, the alarm estimation problem can be presented as equation (3):

$$
\text { alarm }=\operatorname{Pr}(a) \operatorname{Pr}(l, h, d, p, t, r \mid a)>\text { threshold }
$$

In order to estimate the prior probability, we can use the uniform distribution. The threshold can be used to control the sensitivity of generating an alarm.

For estimating the probability $\operatorname{Pr}(l, h, d, p, t, r \mid a)$, we can assume that the variables $l, h, d, p, t$ and $r$ are independent. Thus, we can write the equation (4):

$$
\begin{gathered}
\operatorname{Pr}(l, h, d, p, t, r \mid a) \approx \\
\mathrm{p}(l \mid a) \mathrm{p}(h \mid a) \mathrm{p}(d \mid a) \mathrm{p}(p \mid a) \mathrm{p}(t \mid a) \mathrm{p}(r \mid a)
\end{gathered}
$$

Depending on the number of data available for training, other assumptions could be made. For example, we could jointly estimate several variables, for example $h, p$ and $l$ jointly. See equation (5).

$$
\operatorname{Pr}(l, h, d, p, t, r \mid a) \approx \mathrm{p}(h, p, l \mid a) \mathrm{p}(d \mid a) \mathrm{p}(t \mid a) \mathrm{p}(r \mid a)
$$

Where $\operatorname{Pr}(l, h, d, p, t, r \mid a)$ represents the conditional probability of having $l, h, d, p, t, r$ when an alarm $a$ has been detected, $\operatorname{Pr}(l, h, p \mid a)$ represents the conditional probability of having $l, h, p$ when an alarm $a$ has been detected, $\operatorname{Pr}(d \mid a)$ represents the conditional probability of having $d$ when an alarm $a$ has been detected, $\operatorname{Pr}(t \mid a)$ represents the conditional probability of having $d$ when an alarm $a$ has been detected and finally, $\operatorname{Pr}(r \mid a)$ represents the conditional probability of having $r$ when an alarm $a$ has been detected. From equation 5, we can observe that $d$, $t$ and $r$ will be indepently estimated while $h, p$ and $l$ will be jointly considered.

\section{B. Inductive Training}

We use an inductive approach that estimates the likelihood function using past observations. That is, several measurements are taken for a child; then, the functions $\mathrm{p}(l, h, d, p, t \mid a)$ and $\mathrm{p}(a)$ are estimated. The main drawback of this approach is the time consuming in the training phase. We denote as $T$ the set of training data, formed by $I$ observations with their respective parameters. Each training data, $T_{i}$, is represented as $\left(a_{i}, l_{i}, h_{i}, d_{i}, p_{i}, t_{i}, r_{i}\right)$, where $i$ can be from 1 to $I$. Several alternatives have been proposed in the literature to estimate $\mathrm{p}(l, h, d, p, t \mid a)$ from $T$ : the histogram method [44], [45], the Bayesian method [46] or the kernel method [47]. Bayesian method is used in this paper. 


\section{Model verification}

In order to perform the experimental study, our system was logging data during 6 weeks. $92 \mathrm{~K}$ training data samples were logged. The patient was a 9-year-old child suffering from severe asthma attacks. The data obtained during this period were manually labeled.

The set of data obtained has been divided in two sets: for the training corpus, we used the first 4 weeks, and for the test corpus, we used the last 2 week.

In order to evaluate the proposed model, we prepared next experiment. We use the training corpus to estimate the statistical model. For each observation in the test corpus, the system predicts if an alarm occurs and compares it with the label. Then, we measure the percentage of correct predictions and the recall. Fig. 3 shows the results.

We evaluate five alternative models:

- (a) assumes that the variables are independent; it uses equation (4);

- (b) $h, p$, and $l$ are jointly estimated, it uses (5);

- (c) uses equation (5), where $\mathrm{p}(t \mid a)$ is removed;

- (d) uses equation (5) removing $\mathrm{p}(d \mid a)$ and,

- (e) uses equation (5) removing $\mathrm{p}(r \mid a)$.

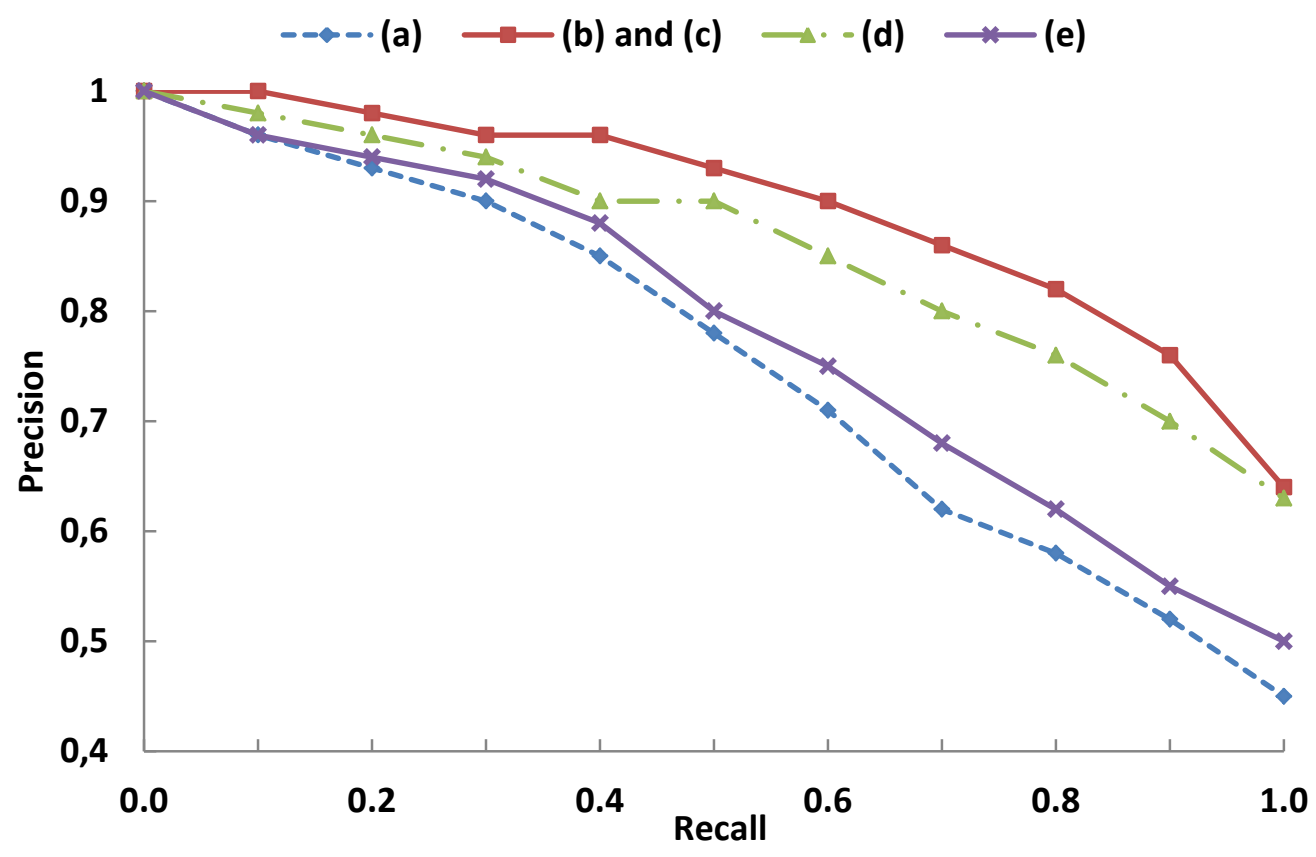

Fig. 3. Evaluation of stochastic alarm prediction model

Form Fig. 3, we can observe that (a) obtains poor results. It is better to estimate jointly the variables when we have enough data and they are related. We tested several combinations of joint variables. The only one that gets a significant improvement is $\mathrm{p}(h, p, l \mid a)$, used in (b). (c) obtains the same results than (b). This indicates that the temperature does not seem to be relevant information in this problem. Observing the data, we can see that (d) which does consider the temporal parameter d, the results are worse. This is because depending on the person, the attacks of chronic asthma usually happens in certain moments of day.. This is reason because we need to train our prediction model. Finally, (e) shows that the abnormal respiration indicator is crucial to the system.

Another experiment has been developed to study the influence of training test size in the performance. We have trained the model using an incremental number of samples, from $10 \mathrm{~K}$, to $60 \mathrm{~K}$. This test has been developed using maximum recall. We used the model described in equation (5), which has obtained the best results in the previous experiment. Fig. 4 shows the results. 


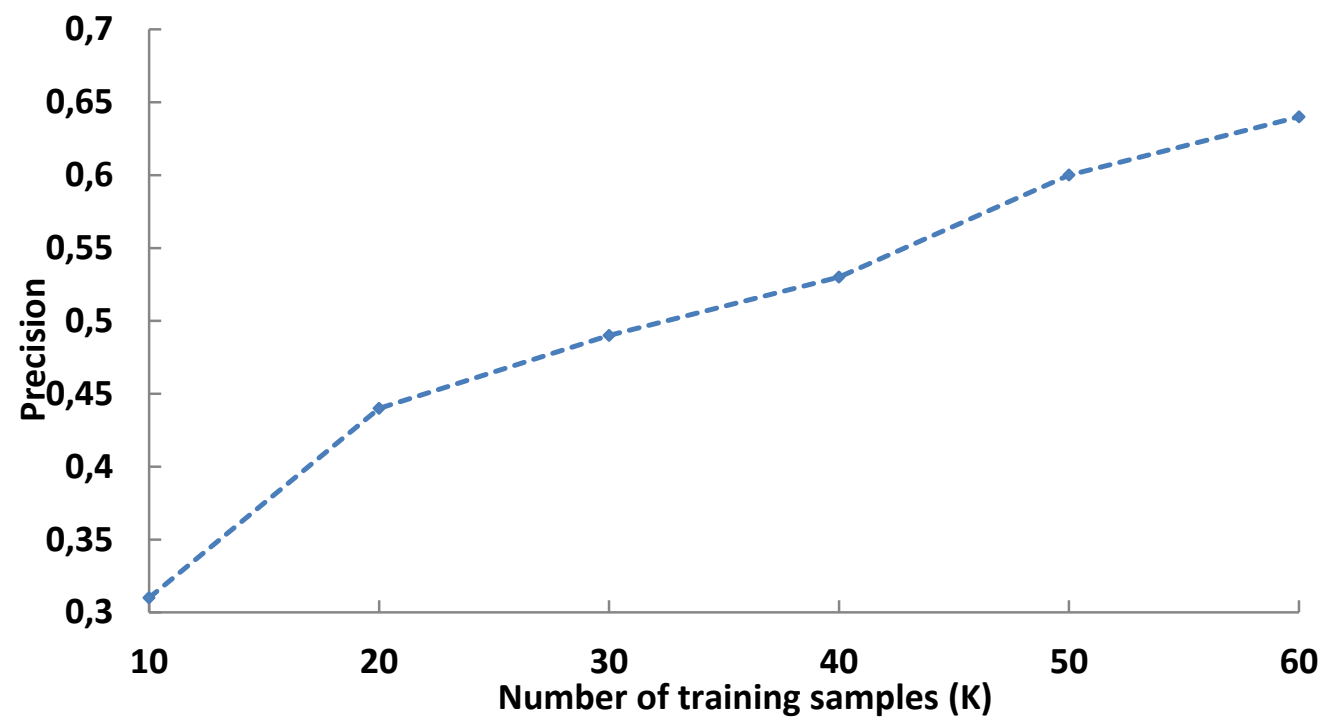

Fig. 4. Evaluation of alarm precision vs size of training corpus

The size of training corpus is directly related with the number of training samples. This kind of systems improves its performance as the training process is longer. Fig. 4 shows the clear influence between the corpus size and the performance. From this graph, we can deduce that an increase of the size of the corpus will lead to an improvement in precision. As we can see, the system achieves a precision of $70 \%$ when the number of samples is around $60 \mathrm{~K}$ samples.

\section{Scenario of Test Bench AND Results}

In order to test our proposed architecture, we have developed a small wireless device to measure the main symptoms that a person suffering a respiratory crisis due to asthma may experience. This device is wirelessly connected to a smartphone via Bluetooth. The smartphone has been connected to the parents' smartphone to transmit the alarm messages. Finally, we have tested the battery evolution of the wearable device to check its autonomy and the consumed bandwidth when data are exchanged between the wearable device and child's smartphone.

\section{A. Developed device}

As Fig. 5 shows, the wearable device is based on a LyliPad Arduino module and a HC-05 Bluetooth interface. It has a diameter of $50 \mathrm{~mm}$ and a thickness of $0.8 \mathrm{~mm}$. The Bluetooth interface is used to connect with the smartphone. As sensors, we have selected a DS18B20 digital thermometer to measure the corporal temperature and an optical sensor which is able to measure the heart beats through the photoplethysmography effect. The number of breathing is measured using an elastic chest band. The location can be defined using the 3G/4G coverage of the child smartphone, instead of using the Global Positioning System (GPS). Finally, through a motion sensor which can be an accelerometer, it is possible to measure the physical activity of the monitored child. 


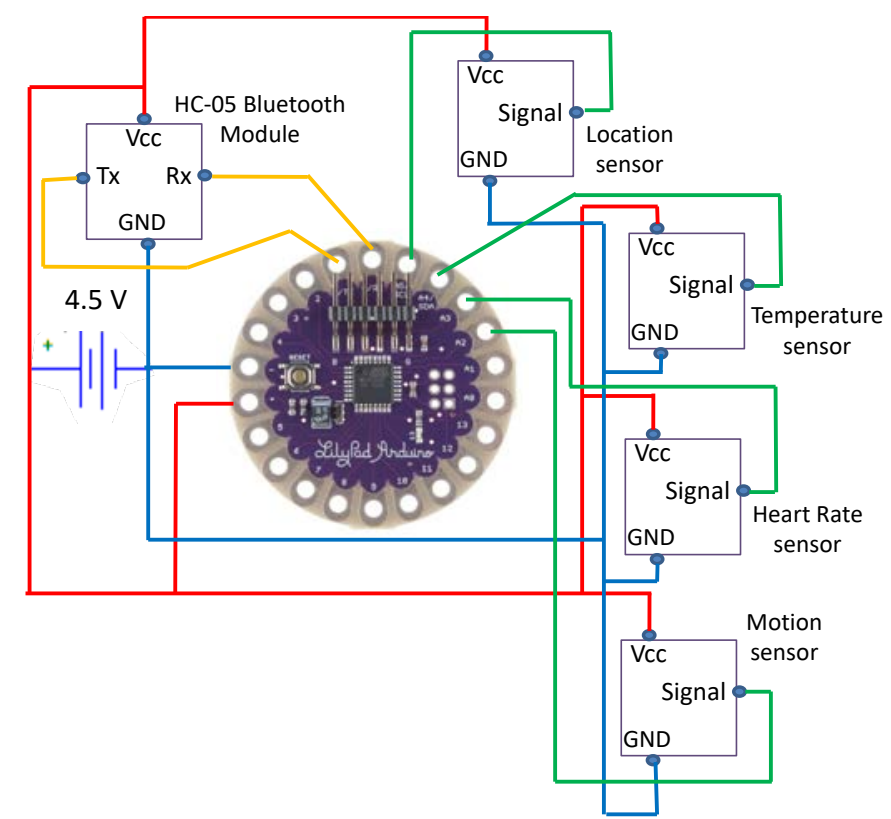

Fig. 5. Wearable device developed to test the proposed architecture

There are some other sensors than can be easily included in this kind of systems in order to detect other symptoms or illness.

For example, the photoplethysmography effect can also be used to measure the blood oxygen saturation. Breathing, sweating and motion are 3 parameters that are usually related. An abnormal value means that something is happening.

To measure the sweating, we could base our measures with the skin conductance level (SCL). In order to monitor this parameter we only need to put 2 metallic electrodes in touch with the skin. When the SCL increases, the level of sweating also increases. This change can be experienced due to practicing some physical activity or because the child is suffering some symptom.

\section{B. Packet structure for data fusion}

As Fig. 5 shows, our system receives information from several sensors. This information is transmitted via Bluetooth to a smartphone that will be in charge of sending this information to the parents.

The data can be merged into different processes, i.e., during the transmission between the LilyPad module and the smartphone or during transmission from the child's smartphone to the parent's device. We decided to perform the data fusion in the first transmitting process.

To do this, the Lilypad device encapsulates information from each sensor in a frame. This frame contains information about the patient (Sender ID), the time the sample was taken (Time of sample), the day (Date), the sensor ID (Sensor ID), type sensor (Typ. Sensors) and finally the data collected (Data from sensor). We need to know the type of sensor and identify it because the same person can carry several sensors of the same type, as could be the case of sensors such as accelerometers.

Because data is transmitted under Bluetooth technology, we must take into account its own structure [48]. Fig. 6 shows the structure of the packets as well as the number of bits assigned to each field. 


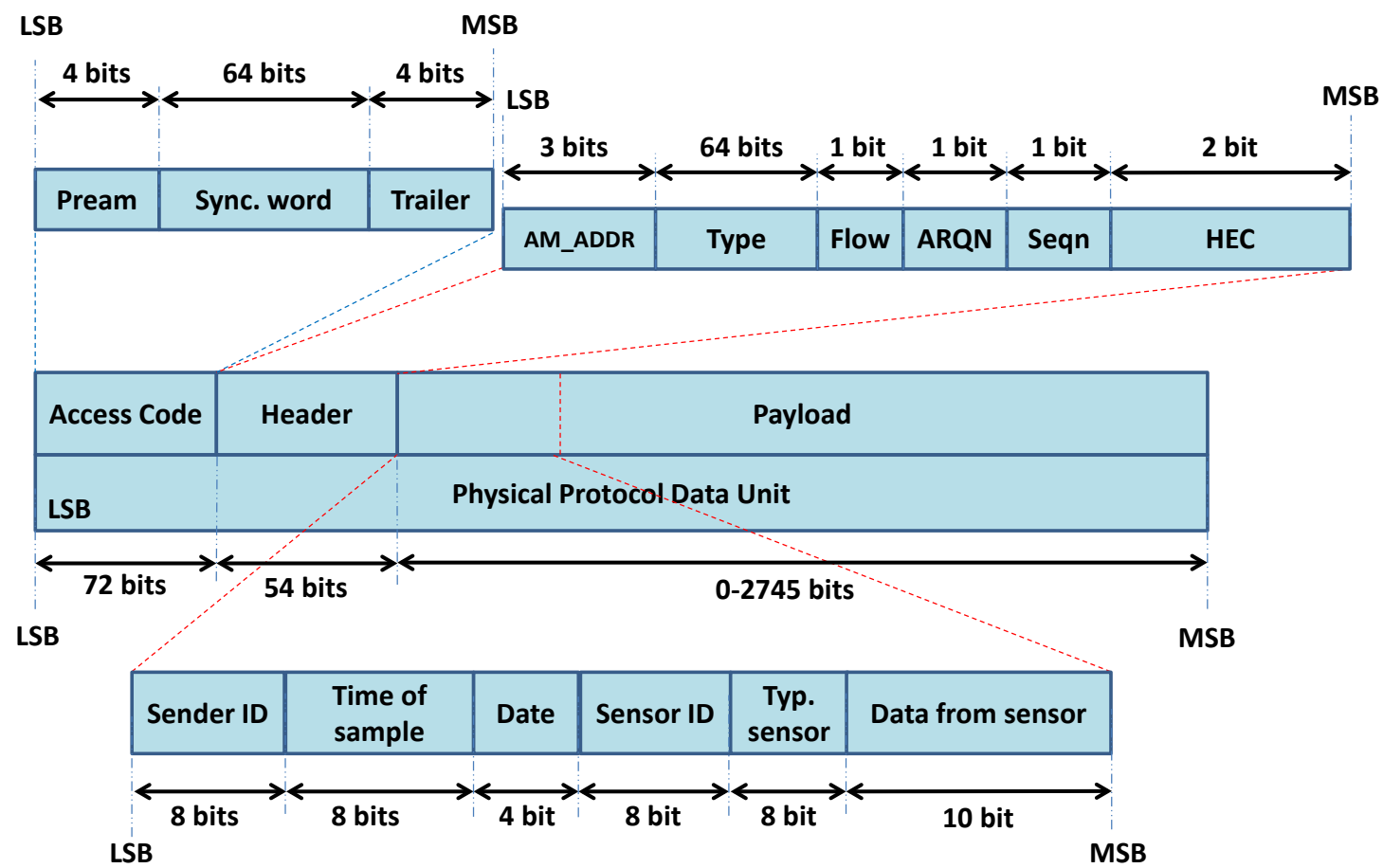

Fig. 6. Packet stucture of each sensor.

In order to perform the information fusion, we start from the basic idea that the measurements are synchronously taken and therefore all the measurements carry the same information regarding the patient, time of sample and date. The Lilypad module is responsible for taking all frames from the sensors and combining their contents, forming a combined super-frame. Fig. 7 shows the data fusion process.

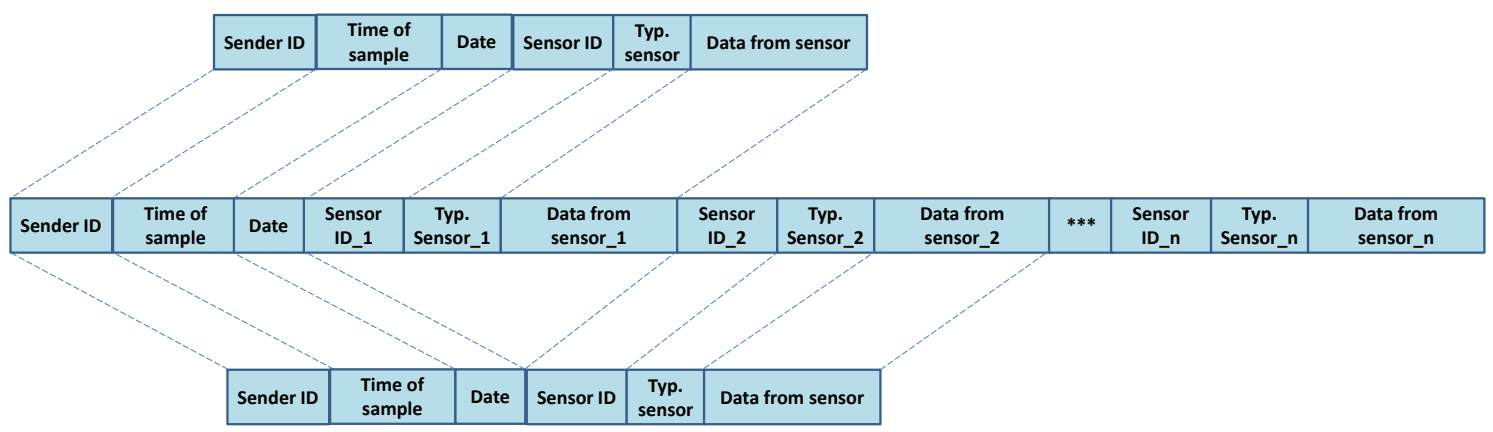

Fig. 7. Data fusion process.

Finally, Fig. 8 shows the packet structure sent from the Lilypad module to the child's smartphone.

\begin{tabular}{|c|c|c|c|c|c|c|c|c|c|c|c|}
\multicolumn{10}{c|}{ Payload (up to 2745 bits) } \\
$\begin{array}{c}\text { Access } \\
\text { Code }\end{array}$ & Header & $\begin{array}{c}\text { Sender } \\
\text { ID }\end{array}$ & $\begin{array}{c}\text { Time of } \\
\text { sample }\end{array}$ & Date & $\begin{array}{c}\text { Sensor } \\
\text { ID_1 }\end{array}$ & $\begin{array}{c}\text { Typ. } \\
\text { Sens_1 }\end{array}$ & $\begin{array}{c}\text { Data } \\
\text { sensor 1 }\end{array}$ & $\begin{array}{c}\text { Sensor } \\
\text { ID_2 }\end{array}$ & $\begin{array}{c}\text { Typ. } \\
\text { Sens_2 }\end{array}$ & $\begin{array}{c}\text { Data } \\
\text { sensor 2 }\end{array}$ & $\ldots$ \\
\hline \multicolumn{10}{|c|}{ Physical Protocol Data Unit } \\
\hline
\end{tabular}

Fig. 8. Combined super-frame packetized inside a Bluetooth packet.

To demonstrate the advantages of performing the information fusion before sending it, we will compare 3 important parameters to evaluate the protocol performance. These are the protocol overhead, protocol efficiency and the amount of information needed to send between both devices. Two cases are considered; 
the first one considers that information is individually transmitted from sensors without performing the data fusion. The second case considers that the module performs the data fusion before transmitting data.

Fig. 9 and Fig. 10 show the protocol overhead and protocol efficiency as a function of number of sensors for both cases, respectively. As we can see, both parameters remain constant when no information fusion is performed. This is due to packets are individually sent and consequently the protocol performance should be calculated for each new packet. On the other hand, when data fusion is performed, we are able to reduce the number of bits from mandatory fields such as mandatory fields and sender ID, date, etc. In this way, we can say that the packets are efficiently used because we try to fill up the field of payload, reducing the amount of useless bits due to headers and other fields only necessary to stablish the communication and ensure that a paper reaches the destination.

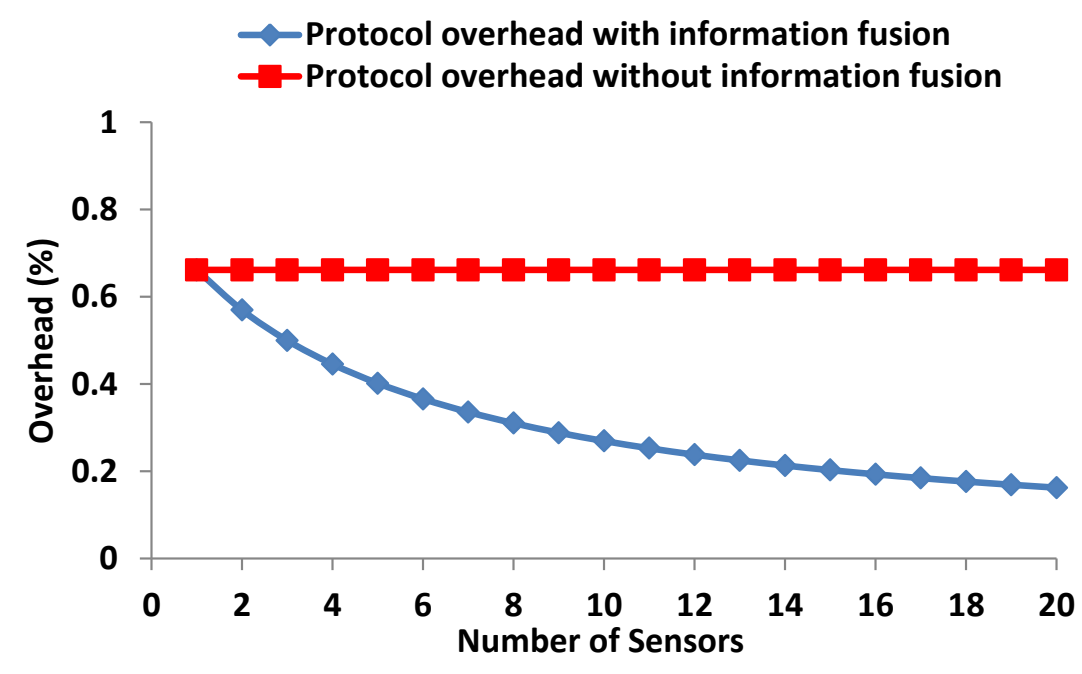

Fig. 9. Protocol overhead.

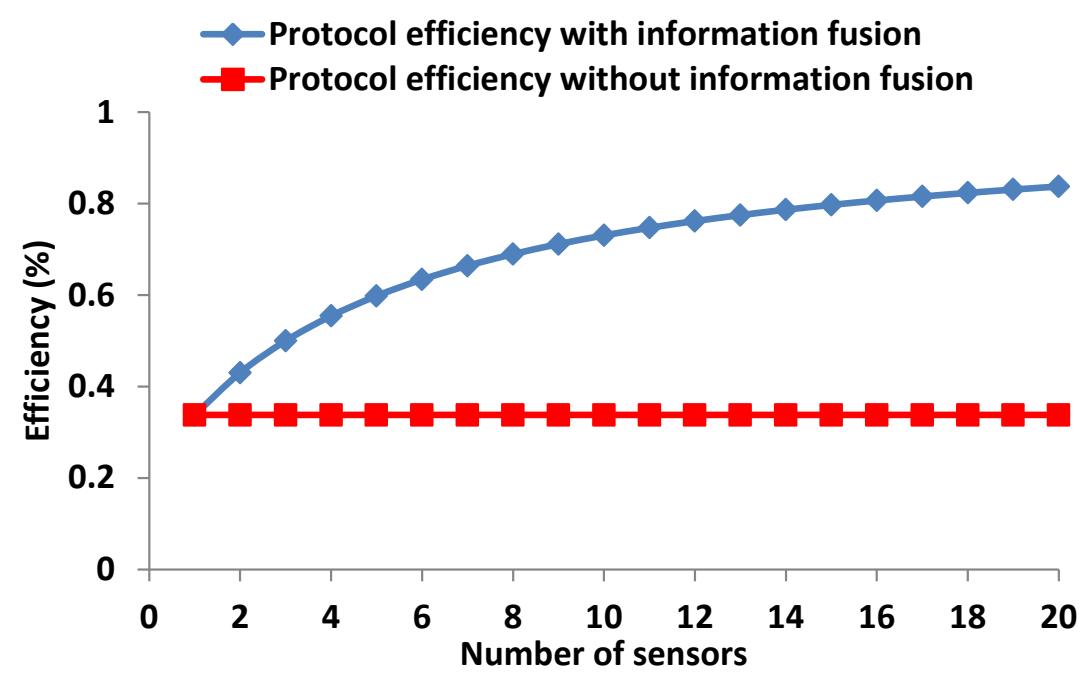

Fig. 10. Protocol efficiency.

Finally, knowing the packet structure, it is easy to determine the amount of bits we need to transmit the sensor information. Fig. 11 shows the amount of bits we would transmit as a function of number of available sensors. In both cases, we observe that the amount of bits transmitted increases in both cases but the slope with which it increases is lower for the case of data fusion is performed. 


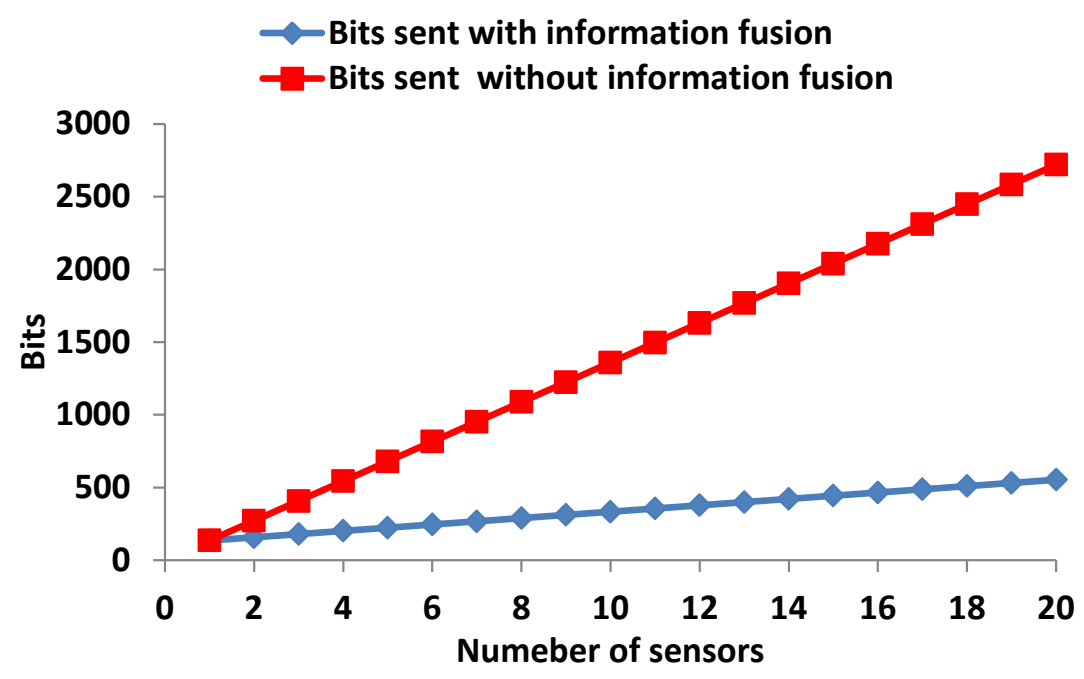

Fig. 11. Amount of bits trnasmitted.

\section{Message exchanged between devices}

One of the most important steps in the communication process is the Bluetooth connection between the wearable device and the smartphone. This connection should be reliable at the time of ensuring the data security for avoiding vulnerabilities. For this reason, after wearable device has sent the request to connect with the child's smartphone, both devices should pair using a pin code. A request to connect is sent when there are data available, i.e., every 30 minutes to send the normal packets, every 24h to send the summary report or when an emergency situation has been detected. Both devices should negotiate the encryption method they are going to use for exchanging data. After that, the wearable device can start sending data. The kind of packed exchanged will vary as a function of the sort of data. In any case, when the device finishes the sending process, it sends a message of requesting to disconnect and waits for the smartphone confirmation. Fig. 12 shows the packet exchange between the three main components of this architecture.

As we can see, the smartphone is in charge of transmitting data to the parents and to the monitoring server. When the parents receive the data, they check if abnormal values have been registered. When something wrong has been detected, parents will push to the smartphone to send the alarm messages. The first one is sent to the monitoring server in order to inform it that it should send the corresponding data to the physician. The message from parents to the physician is automatically sent after the alarm message has been sent to the monitoring server. Finally, the physician will receive the data from the monitoring server. These data will be the sensors information of the current sample and the immediately previous values stored in the monitoring server.

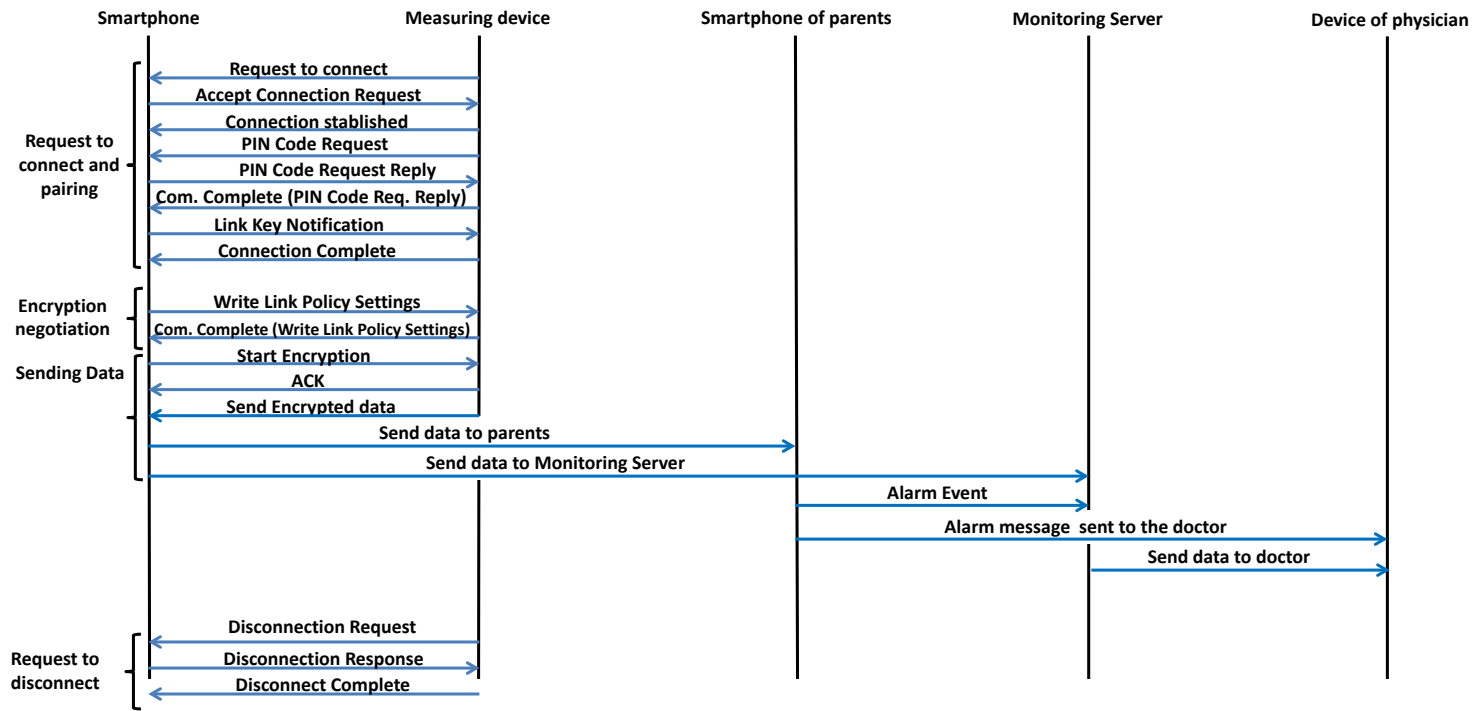

Fig. 12. Packet exchange between the three main components of this architecture 


\section{Andorid App.}

To gather data, we have developed an intuitive and friendly application for android devices. It allows monitoring several parameters such as heart rate, breathing physical activity, temperature and location (Fig. 13a). The results of the measured parameters are collected in a Log file. Fig. 13b shows the configuration screen When some abnormal value is detected the App will show a red signal. Finally, it is possible to access to the location in a map directly pushing on the pink icon.

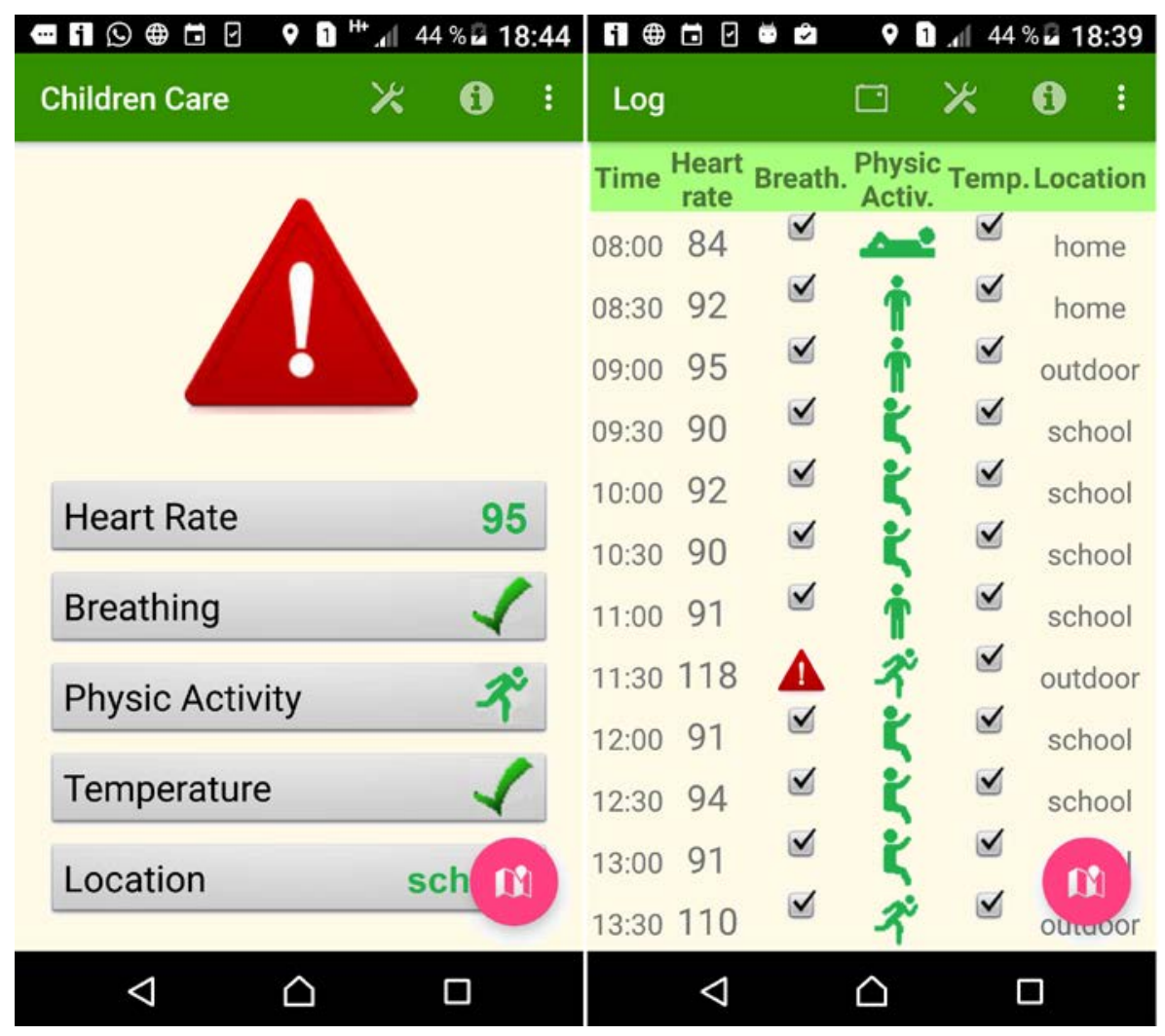

a

Fig. 13. Main screens of the Android application

\section{E. Results}

To check the correct operation of our device, we have connected three different sensors: a heart rate sensor, a temperature sensor and a shock sensor. We have measured the energy consumption during a normal use to check how many hours it can be working without problem (see Fig. 14). It has a battery of $4.5 \mathrm{~V}$ and a capacity of $850 \mathrm{mAh}$. We would be able to continuously work with this system during $33 \mathrm{~h}$.

Finally, Fig. 15 shows the consumed bandwidth in bytes per second (Bytes/s) between both devices. In this case, we observe that the biggest bandwidth is consumed during the connection and disconnection process with values higher than 1000 Bytes/s. The data transmission consumes little bandwidth with values near to 50 Bytes/s. 


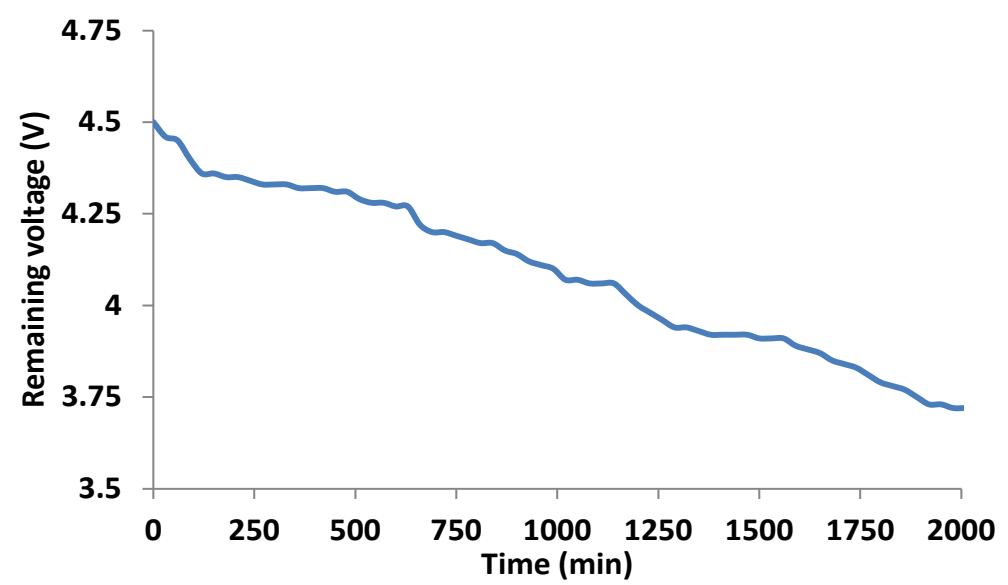

Fig. 14. Battery evolution as a function of time.

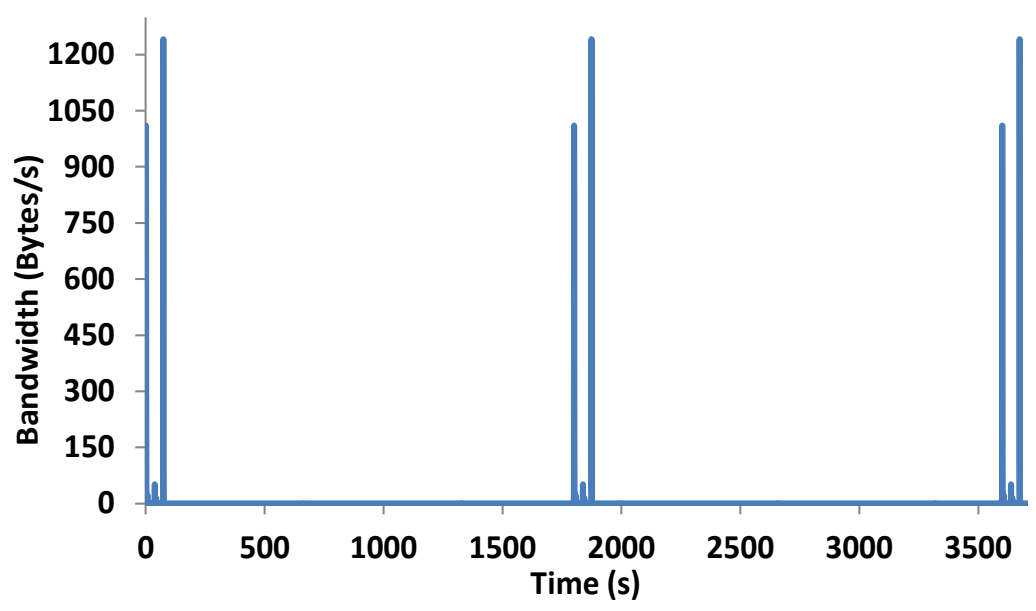

Fig. 15. Consumed bandwidth by wearable device.

\section{CONCLUSION AND FUTURE WORK}

The design and development of low cost smart systems for e-health is one of the main challenges of the $21^{\text {st }}$ century. The monitoring of chronic diseases, especially in children, can help patients and caregivers to improve their own welfare. In this paper, we have presented an intelligent architecture specially designed to monitor children that suffer some chronical illness.

The system is based on wearable sensors and a smartphone that continuously monitors the child activity and vital signs. If some abnormal value is detected the systems sends a set of messages to teachers or parents and to the physician in charge of treating this child. Finally, the system stores the data gathered in a DB. This information is also used to discriminate between real emergency situations and cases of false positives. To implement this, the measurement system is based on a stochastic approach for multi-sensor fusion decision algorithm. In order to carry out the data fusion, we have grouping different analyzed parameters in order to see which parameters are more significant. As results show, in asthma attacks, parameters such as heart rate, breathing, physical activity and time of day are the most important ones and offer better accuracy than other models that consider, for example, the body temperature.After the appropriate training, the system improves the accuracy in their detections. Moreover we have included data fusion on the communication protocol in order to decrease the power consumption. Using this kind of technology, we can improve the medical attention and decrease the number of children hospitalization or shorten hospital stays.

As future work, we want to test our system with many patients in a hospital. In addition, we are also analyzing the possibility of adding this smart system as a part element of a big and secure medical infrastructure [39] to monitor patients in hospitals and disabled and elderly people inside the Ambient Assisted Living (AAL) paradigm [42] [43]. 
This work has been partially supported by the "Ministerio de Educación, Cultura y Deporte", through the "Ayudas para contratos predoctorales de Formación del Profesorado Universitario FPU (Convocatoria 2014)”. Grant number FPU14/02953.

\section{REFERENCES}

[1] J. M. Perrin, S. R. Bloom, S. L. Gortmaker, The increase of childhood chronic conditions in the United States, Jama 297 (24) (2007) 2755-2759.

[2] J. M. Perrin, L. E. Anderson, J. Van Cleave, The rise in chronic conditions among infants, children, and youth can be met with continued health system innovations, Health Affairs 33 (12) (2014) 2099-2105.

[3] J. Van Cleave, S. L. Gortmaker, J. M. Perrin, Dynamics of obesity and chronic health conditions among children and youth, Jama 303 (7) (2010) 623-630.

[4] J. E. Jordan and R. H. Osborne, Chronic disease self-management education programs: challenges ahead, Medical Journal of Australia 186 (2) (2007) 84

[5] M. Ng, T. Fleming, M. Robinson, B. Thomson, N. Graetz, C. Margono, et al., Global, regional, and national prevalence of overweight and obesity in children and adults during 1980-2013: a systematic analysis for the Global Burden of Disease Study 2013, The Lancet 384 (9945) (2014) 766-781.

[6] C. S. Camfield, P. R. Camfield, P. J. Veugelers, Death in children with epilepsy: a population-based study. The Lancet 359 (9321) (2002) 1891-1895.

[7] V. Nodhturft, J. M. Schneider, P. Herbert, Chronic disease self-management, Nurs Clin North Am 35 (2000) 507-518.

[8] A. C. Modi, A. L. Pai, K. A. Hommel, K. K. Hood, S. Cortina, M. E. Hilliard, S. M. Guilfoyle, W. N. Gray, D. Drotar, Pediatric self-management: a framework for research, practice, and policy, Pediatrics 129 (2) (2012) 473-485

[9] H. Alemdar and C. Ersoy, Wireless sensor networks for healthcare: A survey, Computer Networks 54 (15) (2010) 2688-2710.

[10] L. Parra, S. Sendra, J. M. Jiménez, J. Lloret, Multimedia sensors embedded in smartphones for ambient assisted living and ehealth, Multimedia Tools and Applications, (2015) 1-27.

[11] S. Sendra, E. Granell, J. Lloret, J. J. Rodrigues, Smart collaborative system using the sensors of mobile devices for monitoring disabled and elderly people. In Proccedings of the 2012 IEEE International Conference on Communications (ICC), Ottawa, Canada, 10 - 15 Jun 2012, pp. 6479-6483.

[12] K. Lorincz, B. R. Chen, G. W. Challen, A. R. Chowdhury, S. Patel, P. Bonato, M. Welsh, Mercury: a wearable sensor network platform for high-fidelity motion analysis. In proc. of the Proceedings of the 7th ACM Conference on Embedded Networked Sensor Systems (SenSys ’09). Berkeley, California , November 4 - 6, 2009. 183-196.

[13] E. S. Sazonov, G. Fulk, J. Hill, Y. Schutz, R. Browning, Monitoring of posture allocations and activities by a shoe-based wearable sensor, IEEE Transactions on Biomedical Engineering 58 (4) (2011) 983-990.

[14] J. Lloret, S. Sendra, J. M. Jimenez, L. Parra, Providing security and fault tolerance in P2P connections between clouds for mHealth services, Peer-to-Peer Networking and Applications (2015) 1-18.

[15] S. Bergströma, G. Bomanb, L. Erikssone, H. Formgrena, T. Foucardc, L. Hörted, C. Jansonb, U. Spetz-Nyströmb, G. Hedlina, Asthma mortality among Swedish children and young adults, a 10-year study, Respiratory medicine 102 (9) (2008) 1335-1341.

[16] J. N. Germann, D. S. Kirschenbaum, B. H. Rich, Child and parental self-monitoring as determinants of success in the treatment of morbid obesity in low-income minority children, Journal of Pediatric Psychology 32 (1) (2007) 111-121.

[17] S. Sendra, E. Granell, J. Lloret, J. J. Rodrigues, Smart collaborative mobile system for taking care of disabled and elderly people, Mobile Networks and Applications 19 (3) (2014) 287-302.

[18] A. Pantelopoulos and N. G. Bourbakis, A survey on wearable sensor-based systems for health monitoring and prognosis, IEEE Transactions on Systems, Man, and Cybernetics, Part C (Applications and Reviews) 40 (1) (2010) 1-12

[19] N. D. Lane, E. Miluzzo, H. Lu, D. Peebles, T. Choudhury, A. T. Campbell, A survey of mobile phone sensing, IEEE Communications magazine 48 (9) (2010) 140-150.

[20] M. T. Nkosi, F. Mekuria, S. H. Gejibo, Challenges in mobile bio-sensor based mHealth development, In proc. of the 13th IEEE International Conference on e-Health Networking Applications and Services (Healthcom 2011) Columnbia, MO, USA. June 13-15, 2011. Pp. 21-27.

[21] S. Iyengar, F. T. Bonda, R. Gravina, A. Guerrieri, G. Fortino, A. L. Sangiovanni-Vincentelli, A framework for creating healthcare monitoring applications using wireless body sensor networks. In Proc. of the ICST 3rd international conference on Body area networks (BodyNets '08). Tempe, Arizona, March 13 - 17, 2008

[22] G. Fortino, S. Galzarano, R. Gravina, W. Li, A framework for collaborative computing and multi-sensor data fusion in body sensor networks. Information Fusion, 22 (2015) 50-70

[23] R. Gravina, P. Alinia, H. Ghasemzadeh, G. Fortino, Multi-sensor fusion in body sensor networks: State-of-the-art and research challenges. Information Fusion 35 (2017) 68-80.

[24] R. Gravina, and G. Fortino, Automatic Methods for the Detection of Accelerative Cardiac Defense Response. IEEE Trans. Affective Computing 7(3) (2016) 286-298

[25] S. Galzarano, R. Giannantonio, A. Liotta, G. Fortino, A Task-Oriented Framework for Networked Wearable Computing. IEEE Trans. Automation Science and Engineering 13(2) (2016) 621-638.

[26] G. Fortino, R. Giannantonio, R. Gravina, P. Kuryloski, R. Jafari, Enabling Effective Programming and Flexible Management of Efficient Body Sensor Network Applications. IEEE Trans. Human-Machine Systems 43(1) (2013) 115-133.

[27] J. Dai, X. Bai, Z. Yang, Z. Shen, D. Xuan, Mobile phone-based pervasive fall detection, Personal and ubiquitous computing 14 (7) (2010) 633-643

[28] J. Bravo, J. Fontecha, F. J. Navarro, R. Hervas, A mobile proposal for frailty monitoring by rehabilitation and physical daily activity. In Proc. of the 2011 IEEE International Conference on Consumer Electronics (ICCE 2011), Las Vegas, USA, January $9-12$, 2011. 176-180. 
[29] H. Ketabdar, M. Lyra, System and methodology for using mobile phones in live remote monitoring of physical activities, In Proc. of the 2010 IEEE International Symposium on Technology and Society, New South Wales, Australia, June $7-9$, 2010. 350-356.

[30] J. V. Lee, Y. D. Chuah, K. T. Chieng, Smart elderly home monitoring system with an android phone, Int. J. Smart Home 7 (3) (2013) 17-32.

[31] Y. D. Lee and W. Y. Chung, Wireless sensor network based wearable smart shirt for ubiquitous health and activity monitoring, Sensors and Actuators B: Chemical 140 (2) (2009) 390-395.

[32] S. González-Valenzuela, M. Chen, V. C. Leung, Mobility support for health monitoring at home using wearable sensors, IEEE Transactions on Information Technology in Biomedicine 15 (4) (2011) 539-549.

[33] M. Aminian and H. R. Naji, A hospital healthcare monitoring system using wireless sensor networks, J. Health Med. Inform 4 (2013) 121.

[34] A. Bourouis, M. Feham, A. Bouchachia, Ubiquitous mobile health monitoring system for elderly (UMHMSE), arXiv preprint arXiv:1107.3695 (2011).

[35] V. Gay and P. Leijdekkers, A health monitoring system using smart phones and wearable sensors, International journal of ARM 8 (2) (2007) 29-35.

[36] O. Postolache, P. S. Girão, M. Ribeiro, M. Guerra, J. Pincho, F. Santiago, A. Pena, Enabling telecare assessment with pervasive sensing and Android OS smartphone, In Proc. of the 2011 IEEE International Symposium on Medical Measurements and Applications(MeMeA 2011), Bari, Itali, May 30 - 31, 2011.288-293.

[37] P. Leijdekkers, V. Gay, F. Lawrence, Smart homecare system for health tele-monitoring, In Proc. of the 1st International Conference on the Digital Society (ICDS 2007), Guadeloupe, French Caribbean, January 2 - 6, 2007.

[38] K. Lorincz, B-R. Chen, G. W. Challen, A. R. Chowdhury, S. Patel, P. Bonato, and M. Welsh, "Mercury: A Wearable Sensor Network Platform for High-Fidelity Motion Analysis", In Proc. of the 7th ACM Conference on Embedded Networked Sensor Systems, Berkeley, California, November 4-6, 2009.Pp. 183-196.

[39] J. Lloret, A. Canovas, S. Sendra, L. Parra, A smart communication architecture for ambient assisted living, IEEE Communications Magazine 53 (1) (2015) 26-33.

[40] Y. Guo and Bai, G. A General Architecture For Developing A Sustainable Elderly Care E-Health System, International Journal of Information Technology and Business Management (JITBM) 27 (1) (2014), 95-101

[41] M. P. Rajasekaran, S. Radhakrishnan, P. Subbaraj, Elderly patient monitoring system using a wireless sensor network, Telemedicine and e-Health 15 (1) (2009) 73-79.

[42] A. A. Bielskis, V. Denisovas, D. Drungilas, G. Gricius, O. Ramašauskas, Modelling of intelligent multi-agent based E-Health care system for people with movement disabilities, Elektronika ir elektrotechnika 86 (6) (2015) 37-42.

[43] M. Wise, D. H. Gustafson, C. A. Sorkness, T. Molfenter, A. Staresinic, T. Meis, R. P. Hawkins, K. K Shanovich, N. P. Walker, (2007). Internet telehealth for pediatric asthma case management: integrating computerized and case manager features for tailoring a Web-based asthma education program. Health promotion practice, 8(3), 282-291.

[44] D. Gustafson, M. Wise, A. Bhattacharya, A. Pulvermacher, K. Shanovich, B. Phillips, E.Lehman, V. Chinchilli, R. Hawkins, J. S. Kim, The effects of combining Web-based eHealth with telephone nurse case management for pediatric asthma control: a randomized controlled trial, Journal of medical Internet research 14 (4) (2012) 101.

[45] T. Roos, P. Myllymäki, H. Tirri, P. Misikangas, J. Sievänen, A probabilistic approach to WLAN user location estimation. International Journal of Wireless Information Networks, vol. 9 (3) (2002) 155-164.

[46] H. Xie, E, Tanin, L. Kulik, Distributed Histograms for Processing Aggregate Data from Moving Objects. 2007 International Conference on Mobile Data Management, Mannheim, (2007) 152-157.

[47] B. Krishnamachari, S. Iyengar, Distributed Bayesian algorithms for fault-tolerant event region detection in wireless sensor networks. IEEE Transactions on Computers, vol. 53 (3) (2004) 241-250.

[48] X. Nguyen, M. Jordan, B. Sinopoli, A kernel-based learning approach to ad hoc sensor network localization. ACM Transactions on Sensor Networks, vol. 1 (2005) 134-152.

[49] IEEE Std 802.15.1-2002 - IEEE Standard for Information technology - Telecommunications and information exchange between systems - Local and metropolitan area networks - Specific requirements Part 15.1: Wireless Medium Access Control (MAC) and Physical Layer (PHY) Specifications for Wireless Personal Area Networks (WPANs). Available at: http://ieeexplore.ieee.org/iel5/8762/27762/01237559.pdf\&usg=AFQjCNFKax4NQK552-Ybm1uNRZIz3zljCQ. [Last access: April 10, 2017]. 